ENFOQUES JURÍDICOS

REVISTA MULTIDISCIPLINAR DEL CEDEGS

ISSN 26832070

Número 04

julio-diciembre 2021
Artículo: "La radicalización como element vertebrador de la política antiterrorista: una aproximación crítica"

María del Valle Cabeza Domínguez

Carlos Fernández Abad

DOI: https://doi.org/10.25009/ej.v0i04.2568

\title{
La radicalización como elemento vertebrador de la política antiterrorista: una aproximación crítica
}

Recibido 23 febrero 2021-Aceptado 19 marzo 2021.

\author{
Carlos Fernández Abad* \\ Universidad Rey Juan Carlos. Madrid, España \\ carlos.abad@urjc.es \\ María del Valle Cabezas Domínguez** \\ Universidad Rey Juan Carlos. Madrid, España \\ mv.cabezas.2017@alumnos.urjc.es
}

RESUMEN: Los autores de este articulo examinan la validez de la radicalización para constituirse como uno de los elementos vertebradores de la lucha contra el terrorismo de inspiración yihadista en la actualidad. En este sentido, se argumenta que no solo se trata de un concepto problemático y vacío de contenido sino que, además, su naturaleza inespecífica dificulta enormemente la implementación de políticas públicas eficientes para prevenir la participación del individuo en el fenómeno terrorista. Por todo ello, ya en el apartado de
ABSTRACT: The authors of this article address the suitability of the radicalization concept to be one of the vertebral elements of the struggle against jihadist-inspired terrorism. In this sense, it is argued that not only is it a troublesome and empty concept, but also that its non-specific nature makes it enormously difficult to implement efficient public policies to prevent the involvement of the individual in the terrorist phenomenon. For all these reasons, it is claimed the need to subject this concept to a seriously revision

\footnotetext{
* Doctor en Derecho por la Universidad Rey Juan Carlos (Madrid, España) y Doctor en Sociología por la Universidade do Minho (Braga, Portugal). Profesor Ayudante Doctor de Derecho penal y Criminología de la Universidad Rey Juan Carlos.

** Becaria de Colaboración en el Departamento de Derecho Público II y Filología I de la Universidad Rey Juan Carlos, donde realiza una investigación sobre la respuesta del sistema penitenciario español frente a la radicalización yihadista.
}

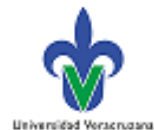


ENFOQUES JURIDICOS

REVISTA MULTIDISCIPLINAR DEL CEDECS

ISSN 26832070

Número 04

julio-diciembre 2021
Artículo: "La radicalización como elemento vertebrador de la política antiterrorista: una aproximación crítica"

María del Valle Cabeza Domínguez

Carlos Fernández Abad

DOI: https://doi.org/10.25009/ej.v0i04.2568 conclusiones, se postula la necesidad de someter a este concepto a una profunda revisión que, por su parte, implique una reformulación de las estrategias antiterroristas contemporáneas.

Palabras clave: Terrorismo yihadista; radicalización; políticas públicas; prevención which, in turn, involves a re-formulation of contemporary anti-terrorist strategies.

Keywords: Jihadist Terrorism; radicalization; public policies; prevention.

SUMARIO: Introducción; 1. Un concepto problemático: ¿qué es la radicalización?; 2. La prevención del terrorismo a partir de la radicalización; Conclusiones; Fuentes de consulta.

\section{Introducción}

La radicalización se constituye actualmente como un concepto hegemónico a la hora de aproximarse al estudio del terrorismo de inspiración yihadista (Antón Mellón y Parra, 2015; Malthaner, 2017; Fernández de Mosteryn y Limón López, 2017). En este sentido, este concepto no solo ha sido ampliamente referido en el mundo académico sino que, por su parte, también ha encontrado una amplia acogida en el ámbito institucional (Sedgwick, 2010), llegando a presentarse como uno de los ejes vertebradores de la lucha contra este fenómeno. En este contexto, según advierten Neumann y Kleinman, la radicalización se habría convertido en una de las palabras de moda de nuestro tiempo (2013: 360), lo que se traduce en una marcada dificultad para encontrar modelos explicativos y planes de intervención que, ya sea de una u otra manera, no orbiten sobre este punto.

Ahora bien, conviene advertir que, a pesar de su incuestionable hegemonía, este concepto ha sido en cierta forma contestado durante los últimos años por un sector todavía minoritario de la literatura especializada, señalándose algunos aspectos como el hecho de que se trata de un concepto ambiguo y falto de contenido (Coolsaet, 2019) o que su mera existencia, además de confundir el activismo político con el terrorismo (Bartlett y Miller, 2010), contribuye a criminalizar a categorías enteras de población (Kundnani, 2010). En este sentido, no resulta sorprendente que hayan surgido numerosos análisis que, enmarcándose en este sector de la doctrina, han criticado la naturaleza del término y, sobre todo, las consecuencias político-criminales que se derivan de su existencia, planteándose en algunos casos la pertinencia de someterlo a una profunda revisión y, en otros, de abandonarlo directamente como principal factor explicativo del terrorismo (Fernández de Mosteryn y Limón López, 2017; Paredes, 2020).

Precisamente, el objetivo principal de este trabajo no es otro que examinar desde una posición crítica la validez de la radicalización para constituirse como el eje vertebrador de la lucha contra el terrorismo en la actualidad. Para su consecución, se plantean una serie de objetivos específicos que se corresponden con el desarrollo del artículo: en primer lugar, se 
ENFOQUES JURIDICOS

REVISTA MULTIDISCIPLINAR DEL CEDECS

ISSN 26832070

Número 04

julio-diciembre 2021
Artículo: "La radicalización como elemento vertebrador de la política antiterrorista: una aproximación crítica"

María del Valle Cabeza Domínguez

Carlos Fernández Abad

DOI: https://doi.org/10.25009/ej.v0i04.2568

aborda el significado del término y los principales problemas que plantea, poniéndose el énfasis en su obligada naturaleza relativa y en la falta de consenso que existe sobre cuál es el punto final de este proceso. Posteriormente, se analiza si, tal y como indican sus partidarios, el marco teórico definido por la radicalización sirve para articular políticas preventivas eficientes en esta materia. Por último, ya en el apartado de conclusiones, se argumenta que el uso de la radicalización como elemento vertebrador de la lucha contra el terrorismo conduce a un adelantamiento de la barrera de intervención que, además de constituirse como una base para la vulneración de derechos fundamentales (Kundnani, 2012), contribuye paradójicamente a crear un escenario más inseguro mediante la implementación de políticas securitarias que resultan altamente lesivas y discriminatorias. En todo caso, más que a la articulación de una definición alternativa del concepto de radicalización -algo que, por su parte, abre una interesante línea de investigación futura-, este texto aspira sobre todo a enarbolar una crítica convincente sobre las desventajas que comporta el uso acrítico de este término, ya sea en la academia o en el campo institucional.

\section{Un concepto problemático: ¿qué es la radicalización?}

Del mismo modo que sucede con el terrorismo, conviene aclarar que no existe un concepto inequívoco del término radicalización (Dalgaard-Nielsen, 2010: 798), siendo este una fuente común de controversias y disputas (Neumann, 2013: 874). En este sentido, la primera cuestión que debe advertirse reside en el hecho de que su uso en el campo de los estudios de terrorismo es en cierta parte novedoso ya que, con anterioridad al año 2001, este era raramente aludido en la literatura especializada y escasamente referido por parte de los medios de comunicación, además de tener una nula representación en las diferentes estrategias antiterroristas (Sedgwick, 2010: 480). Esto, por su parte, no implica en ningún caso que la radicalización sea un concepto totalmente novedoso sino que, más bien, este era empleado en otros contextos diferentes y con un significado distinto. Sobre este punto, Schmid señalada que, durante el siglo XIX y comienzos del XX, muchos partidos políticos se definían a sí mismo como "radicales" para expresar su posición en materias relacionadas con la defensa del republicanismo, el sufragio universal o la democracia (2013: 7). De este modo, como se puede apreciar, la radicalización no va a tener en un primer momento una connotación necesariamente negativa, sino que sobre todo va a estar relacionada con la aspiración de promover profundos cambios sociales de naturaleza progresista (Pisoiu, 2012: 19).

A pesar de que este concepto fue paulatinamente ligado con la violencia durante los años setenta del siglo pasado, especialmente para referirse a los movimientos sociales de extrema izquierda y derecha (Pisoiu, 2012: 21), su presencia en el campo de los estudios de terrorismo durante este periodo fue muy limitada. En su ausencia, predominaban toda una serie de enfoques basados en las "causas profundas" del terrorismo donde se concedía una

pp. $9-26$

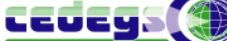


ENFOQUES JURIDICOS

REVISTA MULTIDISCIPLINAR DEL CEDECS

ISSN 26832070

Número 04

julio-diciembre 2021
Artículo: "La radicalización como elemento vertebrador de la política antiterrorista: una aproximación crítica"

María del Valle Cabeza Domínguez

Carlos Fernández Abad

DOI: https://doi.org/10.25009/ej.v0i04.2568

relevancia decisiva a los factores estructurales -fundamentalmente de tipo económico, político y social- que nutren la existencia de este fenómeno (Kundnani, 2015: 14), siendo buena prueba de ellos los análisis realizados por autoras como Crenshaw (1982) o Della Porta (1992). Ahora bien, tras el 11-S, esta discusión sobre las "causas profundas" del terrorismo se tornó especialmente complicada ya que, siguiendo a Kundnani, se consideró que, ante hechos de tal naturaleza, no existía ninguna explicación más allá del fanatismo de los actores implicados, emergiendo como consecuencia otros modelos interpretativos basados en una suerte de ideología del mal que, por su parte, no requería un análisis mucho más profundo que su simple condena y rechazo, otorgándose en este contexto un especial protagonismo a las intervenciones de tipo militar (2012: 48).

A partir del año 2004, sin embargo, este marco interpretativo comenzó a mostrar síntomas evidentes de agotamiento. En este sentido, los atentados de Madrid en 2004 y Londres en 2005 pusieron de relevancia la necesidad de incorporar otros enfoques que permitiesen explicar por qué ciertos jóvenes occidentales aparentemente bien integrados deciden involucrarse en este fenómeno e, incluso, llegar a participar en la planificación y ejecución de actos de terrorismo contra sus países de residencia (Veldhuis y Staun, 2009: 1). La emergencia de la radicalización como principal factor explicativo del terrorismo de inspiración yihadista se situaría precisamente en este punto, estando íntimamente vinculada con la irrupción del "terrorismo doméstico" (Sedgwick, 2010). Es decir, si anteriormente se consideraba que la amenaza que representa este fenómeno provenía de personas y organizaciones extranjeras, ahora se llega a la conclusión de que las personas nacidas y criadas en los países occidentales también pueden involucrarse en el mismo (Crone y Harrow, 2011: 521), lo que estimula la necesidad de examinar las causas que explican por qué algunas personas se adhieren a una visión rigorista y excluyente del credo islámico que, a la postre, puede propiciar la justificación y realización de actos violentos (Kundnani, 2010: 5). Además, nótese que la pretensión de responder a esta pregunta no va a venir únicamente determinada por un mero interés académico sino que los operadores políticos también van a utilizar este concepto para desarrollar numerosos planes de prevención, lo que va a provocar que, desde un inicio, la radicalización adquiera una fuerte dimensión operativa y se institucionalice rápidamente (Fernández de Mosteryn, 2017: 809). Ahora bien ¿en qué consiste exactamente la radicalización?

Como se ha señalado más arriba, en la actualidad no existe un concepto único del término radicalización. En este sentido, Coolsaet señala que, quince años después de su consolidación como centro explicativo del terrorismo, este sigue siendo un concepto ambiguo y mal definido que, en cuantiosas ocasiones, es utilizado de forma vaga e imprecisa (2019: 30), lo que da lugar a la existencia de múltiples interpretaciones sobre su significado. En una línea similar, Fernández de Mosteryn y Limón López advierten que, como objeto de estudio, la radicalización suscita más dudas que acuerdo y no hay una evidencia concluyente 
ENFOQUES JURIDICOS

REVISTA MULTIDISCIPLINAR DEL CEDECS

ISSN 26832070

Número 04

julio-diciembre 2021
Artículo: "La radicalización como elemento vertebrador de la política antiterrorista: una aproximación crítica"

María del Valle Cabeza Domínguez

Carlos Fernández Abad

DOI: https://doi.org/10.25009/ej.v0i04.2568

que permita saber qué es y cómo opera exactamente (2017: 806). Pero ¿por qué es tan difícil llegar a una definición universalmente aceptada de este concepto? Sobre esta cuestión, Neumann señala dos elementos que, de una forma u otra, explican esta dificultad (2013: 873-893):

Por una parte, el autor alemán advierte que, por sí misma, esta palabra no tiene un significado concreto ya que su contenido está necesariamente ligado a lo que se considera como moderado en una sociedad determinada durante un periodo concreto de tiempo. Es decir, la radicalización sería necesariamente un concepto relativo cuyo significado remite a un marco histórico, político y social más amplio, lo que provoca que existan múltiples interpretaciones sobre su alcance y extensión. A modo de ejemplo, Neumann señala que, mientras que la defensa de la libertad de expresión puede ser un principio radical en Corea del Norte, esta es visualizada como un derecho fundamental en la mayor parte de los países occidentales (2013: 876). En este sentido, resulta importante subrayar que, lejos de ser realidades naturales, tanto la radicalidad como la moderación son construcciones sociales que se inscriben siempre en un contexto determinado (Githens-Mazer, 2012; 557), lo que impide que exista un significado universalmente aceptado sobre ambos términos. Además, al margen de esta dificultad, también resulta importante subrayar que trazar una línea divisoria entre la radicalidad y la moderación no siempre es una cuestión tan evidente como parece, con todos los problemas que de ello se derivan (Sedgwick, 2010: 481).

Por otra parte, en relación con el segundo elemento, Neumann señala que tampoco existe un consenso claro sobre cuál debería ser considerado el punto final de este proceso (2013: 874). En estos términos, se abre el interrogante de si únicamente deben ser etiquetados como "radicalizados" aquellos individuos que finalmente participan en la planificación y ejecución de actos violentos o si, en una dimensión más amplia, también deben serlo los que legitiman o justifican el uso de la violencia como medio para la obtención de ciertos objetivos políticos (Richards, 2011: 144). Sobre esta cuestión, la literatura especializada ha tendido a realizar -aunque en numerosas ocasiones de forma confusa y ambigua- una distinción entre la "radicalización cognitiva" y la "radicalización violenta" (Neumann, 2013; McCauley y Moskalenko, 2014; Hafez y Mullins, 2015). Mientras que, por su parte, la primera estaría referida a una disposición creciente a apoyar y perseguir cambios de gran alcance en la sociedad que entran en conflicto con el orden social existente o suponen una amenaza directa para él, la segunda tendría lugar cuando esta pretensión es acompañada del uso o la justificación de medios violentos (Dalgaard-Nielsen, 2010: 798).

En torno a esta distinción entre ideología y comportamiento violento, ha existido un importante debate en la literatura especializada sobre si una conduce necesariamente a la otra (Jenkins, 2007). En este sentido, ha existido una cierta tendencia a considerar la "radicalización cognitiva" como un paso previo a la "radicalización violenta" (Malthaner,

pp. $9-26$

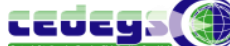


ENFOQUES JURIDICOS

REVISTA MULTIDISCIPLINAR DEL CEDECS

ISSN 26832070

Número 04

julio-diciembre 2021
Artículo: "La radicalización como elemento vertebrador de la política antiterrorista: una aproximación crítica"

María del Valle Cabeza Domínguez

Carlos Fernández Abad

DOI: https://doi.org/10.25009/ej.v0i04.2568

2017: 387). Partiendo de esta premisa, se ha afirmado que las organizaciones radicales no violentas también representan un riesgo para la seguridad ya que, de un modo u otro, estas se constituyen como una suerte de trampolín que estimula la participación futura del individuo en el fenómeno terrorista (Baran, 2005). En este contexto, por ejemplo, Neumann sostiene que un entendimiento completo de la radicalización exige tener en cuenta tanto la parte cognitiva como comportamental del proceso ya que, sin la primera, la segunda no se entiende (2013: 880). En una línea similar, Alonso esgrime que es precisamente la ideología la que aporta los marcos necesarios para que las personas encuadren sus diagnósticos e interpretaciones sobre la realidad, lo que supone que esta adquiera un papel primordial en el proceso de radicalización (2013: 237).

Otros autores, sin embargo, van a negar la existencia de esta relación al tomar como punto de partida que numerosas personas manejan una ideología radical y solo una ínfima parte de ellas termina por involucrarse en el fenómeno terrorista (Bartlett y Miller, 2010; Borum, 2011; McCauley y Moskalenko, 2014). Asimismo, dentro de este sector doctrinal, también se señala que no todas las personas que participan en este fenómeno tienen un conocimiento excesivamente amplio de la ideología que cohesiona a la organización, pudiendo haberse integrado en la misma por otras razones (Borum, 2012: 9). Crone va incluso más allá al sugerir una relación inversa entre ambas variables. Es decir, para este autor, si se toma en consideración que una buena parte de las personas que se involucran en este fenómeno tienen un pasado delictivo (Basra, Neumann y Brunner, 2016), sería la violencia la que se constituye como una precondición para asumir esta ideología y no al revés (2016: 591).

Según apunta Neumann, lejos de ser una cuestión puramente académica, este debate también ha tenido su plasmación en el diseño de las políticas públicas orientadas a luchar contra la radicalización (2013: 885 y ss.): mientras que, por un lado, puede apreciarse la existencia de un enfoque que postula la necesidad de incidir únicamente sobre la "radicalización violenta" al considerar que la "radicalización cognitiva" es perfectamente legítima en el marco definido por una sociedad plural y democrática, también se puede apreciar la presencia de otro modelo alternativo que esgrime la pertinencia de incidir sobre ambos tipos de radicalización. En este sentido, nótese que, en función del enfoque adoptado, se conceden diferentes grados de protagonismo a los agentes implicados en la lucha contra este fenómeno: si, en el primero, la policía y el Derecho Penal desempeñan una posición de centralidad, en el segundo se concede una importancia decisiva a la utilización de otros instrumentos preventivos, como sería el caso de las contranarrativas. Por su parte, la estrategia Prevent en Reino Unido se constituiría como un ejemplo paradigmático en este último sentido ya que, como parte de sus objetivos, se alude a la necesidad de confrontar los idearios que, sin manifestarse abiertamente en favor de la 
ENFOQUES JURIDICOS

REVISTA MULTIDISCIPLINAR DEL CEDECS

ISSN 26832070

Número 04

julio-diciembre 2021
Artículo: "La radicalización como elemento vertebrador de la política antiterrorista: una aproximación crítica"

María del Valle Cabeza Domínguez

Carlos Fernández Abad

DOI: https://doi.org/10.25009/ej.v0i04.2568

violencia, pueden servir de base para el reclutamiento y la radicalización por parte de las organizaciones terroristas (Reinares y García Calvo, 2013).

En definitiva, tal y como se ha podido apreciar a lo largo de este apartado, a pesar de incuestionable hegemonía, el concepto de radicalización presenta al menos dos problemas sustanciales que impiden llegar a una definición aceptada universalmente. Si, por una parte, su contenido está necesariamente ligado a lo que se considerada como moderado en un contexto determinado, por el otro, tampoco existe un consenso claro sobre cuál debería ser considerado el punto final del proceso, existiendo una importante discusión en la literatura especializada sobre la relación entre ideología y comportamiento violento. En este sentido, las definiciones ofrecidas son, en el mejor de los casos, parciales y limitadas. Por ejemplo, mientras que Alonso define la radicalización como "el proceso en función del cual algunos individuos asumen idearios extremistas llegando a aceptar la justificación y realización de acciones violentas en nombre de una causa" (2009: 31), Rabasa y sus colaboradores señalan que esta hace referencia a un proceso basado en la adopción de un sistema de creencias extremistas que, como medio para perseguir un cambio social efectivo, legitima tanto el apoyo como el uso de la violencia (Rabasa, Pettyjohn, Ghez y Boucek, 2010: 1).

Por su parte, conviene notar que, así entendida, la radicalización no es algo exclusivo del yihadismo sino que, en una dimensión más general, también se puede apreciar en otros fenómenos delictivos (Pisoiu, 2012: 11). Ahora bien, resulta importante advertir que, al margen de algunas excepciones (Pérez Cepeda, 2018; Bentley, 2015), la literatura especializada se ha concentrado de forma predominante en esta modalidad de radicalización, empleándose comúnmente términos compuestos como "radicalización yihadista" o "radicalización islamista". Por ejemplo, Reinares y sus colaboradores la definen como la "paulatina asunción, en mayor o menor grado, de actitudes y creencias propias del salafismo, que a través de sus diversas expresiones ofrece desde una visión fundamentalista y excluyente del credo islámico hasta una justificación religiosa del terrorismo" (Reinares, García-Calvo y Vicente, 2019: 67). En el caso de Jordán, este autor define la radicalización yihadista como "el proceso mediante el que el individuo incorpora un sistema de creencias que incluye la voluntad de emplear o apoyar activamente la violencia con el fin de alcanzar los objetivos del salafismo yihadista" (2009: 198).

Una vez examinada la definición de "radicalización" y los problemas que esta plantea, resulta ahora necesario dirigir la atención hacia cómo ha sido utilizado este concepto a la hora de articular políticas públicas en esta materia y, sobre todo, si estas son eficientes para prevenir la participación del individuo en la planificación y/o ejecución de actos de terrorismo. En otras palabras, analizar cómo ha sido operativizado este concepto y, sobre todo, las consecuencias que se derivan de su omnipresencia en las estrategias antiterroristas.

pp. $9-26$

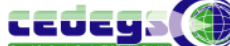


ENFOQUES JURIDICOS

REVISTA MULTIDISCIPLINAR DEL CEDECS

ISSN 26832070

Número 04

julio-diciembre 2021
Artículo: "La radicalización como elemento vertebrador de la política antiterrorista: una aproximación crítica"

María del Valle Cabeza Domínguez

Carlos Fernández Abad

DOI: https://doi.org/10.25009/ej.v0i04.2568

\section{La prevención del terrorismo a partir de la radicalización}

Tal y como se advirtió más arriba, el estudio de la radicalización no ha venido únicamente mediado por un mero interés académico, sino que, a nivel político, este concepto también ha sido utilizado para articular un número significativo de políticas públicas en esta materia, lo que ha propiciado que el concepto adquiera una dimensión operativa y se institucionalice rápidamente (Fernández de Mosteryn y Limón López, 2017: 809). En este sentido, resulta importante notar que, al partir de la consideración de que la participación del individuo en este fenómeno es el producto de una radicalización previa, este marco epistemológico hace posible desarrollar numerosas líneas de intervención antes de que la amenaza llegue a materializarse en la planificación o ejecución de un atentado, residiendo precisamente en este punto uno de sus mayores atractivos (Heath-Kelly, 2012: 5). Es decir, a través de la formulación de toda una serie de indicadores de riesgo, el "discurso sobre la radicalización" ofrecería la posibilidad de intervenir sobre un escenario que todavía no ha sucedido y, por tanto, es desconocido (Mythen, Walklate y Peatfield, 2017: 186).

Ahora bien, antes de analizar si realmente este marco teórico se encuentra en disposición de servir de base para articular políticas públicas que prevengan de forma eficiente la participación del individuo en el fenómeno terrorista, conviene examinar brevemente cuáles son sus fundamentos. En este sentido, numerosos autores han comparado la radicalización yihadista con una suerte de pirámide en la que, mientras que la base está compuesta por un gran contingente de personas que comparten la ideología y los objetivos que dicen perseguir estas organizaciones, la cúspide acogería a un grupo mucho más reducido de individuos que están dispuestos a perseguir tales finalidades mediante el apoyo o el uso de la violencia (McCauley y Moskalenko, 2008; Cano Paños, 2010; Moyano y Trujillo, 2013). Precisamente, para McCauley y Moskalenko, la radicalización no sería otra cosa que ir subiendo a lo largo de esta pirámide (2008: 417). Pero ¿cuáles son los factores que explican el inicio y el desarrollo de estos procesos?

Sobre esta cuestión, tras analizar los cinco modelos dominantes a la hora de aproximarse al estudio de la radicalización (Borum, 2003; Moghaddam, 2005; Wiktorowicz, 2006; Silber y Bhatt, 2007; Sageman, 2008), King y Taylor señalan que todos ellos coinciden en señalar la importancia de dos elementos para explicar por qué ciertos jóvenes occidentales se aproximan a una visión rigorista y excluyente del credo islámico que, a la postre, puede conducir a la planificación y/o comisión de actos relacionados con el terrorismo de inspiración yihadista (2011: 614-615). De un lado, según advierten estos autores, la privación relativa desempeña un papel fundamental en este proceso. Es decir, no se trata de las condiciones materiales experimentadas sino sobre todo de cómo son estas interpretadas, siendo valoradas como el producto de una injusticia. En este sentido, entre las personas que se radicalizan, los sentimientos de enfado y desafección suelen ser comunes, pudiendo provenir de cuestiones como la política exterior de los países

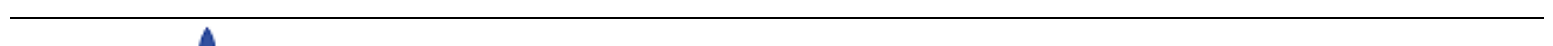


ENFOQUES JURIDICOS

REVISTA MULTIDISCIPLINAR DEL CEDECS

ISSN 26832070

Número 04

julio-diciembre 2021
Artículo: "La radicalización como elemento vertebrador de la política antiterrorista: una aproximación crítica"

María del Valle Cabeza Domínguez

Carlos Fernández Abad

DOI: https://doi.org/10.25009/ej.v0i04.2568

occidentales en el mundo árabe o las situaciones de discriminación vividas por la persona en su país de residencia.

Por otra parte, estos modelos también conceden una importancia decisiva a las crisis de identidad en el desarrollo de los procesos de radicalización. Silber y Bhatt, en una línea muy parecida a la apuntada por Wiktorowicz (2006), hacen referencia a cómo estos procesos suelen ir precedidos de una crisis personal que implica la asunción de una nueva identidad que, por su parte, está muy ligada a una interpretación radical y excluyente del credo islámico (2007: 7). Por otra parte, Sageman también hace alusión a esta cuestión cuando señala en su análisis que la población musulmana en Occidente experimenta frecuentes problemas identitarios (2008: 231). Esto, además, conectaría con el diagnóstico realizado por otros autores (Khosrokhavar, 2003; Roy, 2005; Kepel, 2006) que han señalado que la radicalización tiene lugar frecuentemente entre personas que buscan reconstruir su identidad en un mundo que se percibe como hostil y confuso, algo que sería más frecuente entre las segundas y terceras generaciones de jóvenes inmigrantes que, si bien ya no se sienten parte de la comunidad de sus padres, sufren todo un conjunto de discriminaciones y desventajas económicas en los países occidentales que conducen a un sentimiento de nopertenencia. En este contexto, sería el islamismo militante el que, para algunas personas, ofrece respuestas a la búsqueda de identidad, dignidad y significado (Dalgaard-Nielsen, 2010: 798).

En resumen, la radicalización ha tendido a ser visualizada por parte de la literatura especializada como el resultado de una combinación entre los sentimientos de privación relativa y la concurrencia de ciertos problemas identitarios. En este punto, precisamente, se situaría la noción de vulnerabilidad y cómo esta, en combinación con la presencia de una ideología radical y ciertos factores situacionales -especialmente en lo que se refiere a los lazos sociales previos (Sageman, 2004)-, no hace sino crear las bases adecuadas para que la persona termine participando en el fenómeno terrorista (Heath-Kelly, Baker-Ball y Jarvis, 2015: 6). En este sentido, Cassam esgrime que, desde esta perspectiva, la radicalización ha sido conceptualizada como una suerte de proceso vírico donde la ideología se constituiría como una partícula infecciosa que, a través de diversos soportes -ya sean reclutadores, libros o cualquier otro medio audiovisual-, es inoculada sobre una población que, debido a cómo interpreta su propia situación, se caracteriza sobre todo por su vulnerabilidad (2018: 195).

Una vez examinados los fundamentos teóricos que nutren este marco epistemológico, conviene notar que, a nivel político, estos han estado especialmente presentes en las estrategias antiterroristas contemporáneas, actuando como una suerte de guía que utilizan los poderes públicos para prevenir la aparición de estos procesos o, en su caso, neutralizar su existencia. Es decir, a través de la asunción acrítica que han realizado los diferentes gobiernos de este discurso, se habría pasado desde una definición académica a un uso

pp. $9-26$

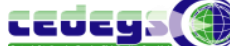


ENFOQUES JURIDICOS

REVISTA MULTIDISCIPLINAR DEL CEDECS

ISSN 26832070

Número 04

julio-diciembre 2021
Artículo: "La radicalización como elemento vertebrador de la política antiterrorista: una aproximación crítica"

María del Valle Cabeza Domínguez

Carlos Fernández Abad

DOI: https://doi.org/10.25009/ej.v0i04.2568

operativo del término. Sobre esta cuestión, sirva como ejemplo que, en el caso español, el denominado Plan Estratégico Nacional de Lucha contra la Radicalización Violenta señala entre otras muchas cuestiones- que su objetivo no es otro que llegar constituirse como un "instrumento eficaz de detección temprana y neutralización de los brotes y focos de radicalismo violento, actuando sobre aquellas comunidades, colectivos o individuos en situación de riesgo o vulnerabilidad" (Gobierno de España, 2015: 5) o que, en todo caso, se buscará "impedir la propagación de ideologías radicales violentas (Gobierno de España, 2015: 8). Ahora bien, ¿realmente sirven estos elementos para articular políticas públicas que, de forma eficiente, prevengan la participación del individuo en el fenómeno terrorista?

En primer lugar, en relación con el valor de la ideología -ya sea la de tipo salafista o cualquier otra que se caracterice por su radicalidad- para anticipar la participación del individuo en la planificación y/o ejecución de actos violentos, resulta importante advertir que, a pesar de muchas personas manejan estos sistemas de creencias, solo una ínfima parte de ellos termina por involucrarse en el fenómeno terrorista (McCauley y Moskalenko, 2008). Es decir, el salafismo no conduce necesariamente al terrorismo de inspiración yihadista (Roy, 2008: 1). En este sentido, la investigación efectuada por Bartlett y Miller (2010) resulta especialmente interesante ya que, desde una perspectiva empírica, los autores examinan cómo se manifiestan las diferentes variables aludidas más arriba en una muestra compuesta por tres grupos de personas diferentes: sujetos relacionados con actividades relacionadas con el terrorismo yihadista, radicales no violentos que se muestran a favor de cuestiones como la implementación de la sharia y, en último lugar, jóvenes musulmanes. En lo que se refiere de forma específica a la ideología, los autores llegaron en sus resultados a la conclusión de que entre el primer y segundo grupo no existían diferencias sustanciales en este punto, aunque sí se podía apreciar en el grupo de los terroristas un conocimiento más simplista y descontextualizado de la religión (Bartlett y Miller, 2010: 9).

En estos términos, al ser una variable compartida por ambos grupos, parece dificil afirmar que, por sí misma, la ideología tiene un valor suficiente como para anticipar la participación del individuo en el fenómeno terrorista y, en consecuencia, articular los programas preventivos sobre este punto. Es decir, como señala Cassam, si se toma en consideración que solo una pequeña proporción de las personas que comparten estas creencias termina participando en hechos violentos, resulta evidente que la "radicalización cognitiva" tiene un escaso poder predictivo (2018: 195). Por otra parte, en referencia a los sentimientos de privación relativa y las crisis de identidad, la situación es muy similar. En este sentido, Lynch advierte que los sentimientos de injusticia no son propiedad exclusiva de los terroristas de inspiración yihadista, sino que, más bien, estos tienen una amplia representatividad entre la población musulmana en general y los jóvenes en particular (2013: 245). De nuevo, el estudio empírico efectuado por Bartlett y Miller resulta esclarecedor ya que, de un modo similar a lo que acontece con el grupo de los terroristas, 
ENFOQUES JURIDICOS

REVISTA MULTIDISCIPLINAR DEL CEDECS

ISSN 26832070

Número 04

julio-diciembre 2021
Artículo: "La radicalización como elemento vertebrador de la política antiterrorista: una aproximación crítica"

María del Valle Cabeza Domínguez

Carlos Fernández Abad

DOI: https://doi.org/10.25009/ej.v0i04.2568

los radicales y los jóvenes musulmanes también experimentan importantes sentimientos de enfado con respecto a cuestiones como la política exterior de los países occidentales o se sienten injustamente tratados por el Estado (2010: 6). Por otra parte, las crisis de identidad tampoco son algo demasiado específico ya que, como señala Mythen, Walklate y Petfield, estas son una condición inherente a la vida humana, especialmente entre los más jóvenes (2017: 191).

De este modo, como se puede apreciar, el marco teórico sobre la radicalización señala toda una serie de factores que no solo están presentes en las personas que se involucran en el fenómeno terrorista, sino que, en una dimensión más amplia, también pueden encontrarse con cierta facilidad en grupos más amplios de población, especialmente entre los jóvenes musulmanes. Este hecho, sin duda, vendría en gran parte motivado por el hecho de que, en la inmensa mayoría de casos, la literatura especializada sobre esta materia no ha utilizado grupos de control en el diseño de sus investigaciones, lo que se constituye como un importante déficit metodológico (Fernández de Mosteryn y Limón López, 2017: 910). Es decir, estas investigaciones suelen focalizarse en el estudio de un número muy limitado de casos y, a partir de los mismos, identificar la concurrencia de toda una serie de variables, pero sin tomar en consideración cómo se manifiestan estas en grupos más amplios de población que nada tienen que ver este fenómeno. En estos términos, cuando sí se establece grupos de control -tal y como hacen, por ejemplo, Bartlett y Miller (2010)-, rápidamente se advierte que cuestiones como el hecho de asumir una ideología radical o experimentar sentimientos de privación relativa y crisis de identidad son más comunes de lo que el "discurso sobre la radicalización" parece sugerir.

Ahora bien, esta dificultad para distinguir entre uno y otro grupo no ha impedido que los poderes públicos asuman de forma acrítica este discurso en la formulación de sus respectivas estrategias antiterroristas, lo que a efectos prácticos ha terminado por traducirse en que la vulnerabilidad sea equiparada con la peligrosidad (Heath-Kelly, 2012: 9), con todas las consecuencias que de ello se derivan. En otras palabras, todas aquellas personas que presentan estas variables serían visualizadas dentro de este marco como potencialmente peligrosas, justificándose de este modo un adelantamiento de la barra de intervención que, por su parte, resulta altamente lesivo desde el punto de vista de la protección de los derechos fundamentales. Esta forma de proceder, por ejemplo, puede apreciarse claramente en la gestión que ha realizado el sistema penitenciario español de los posibles casos de radicalización que pudiesen darse en este medio. En este sentido, con la finalidad de prevenir la aparición de estos procesos, la población penitenciaria considerada como vulnerable -algo que, por su parte, estaría íntimamente conectado con la religiosidad de la persona- ha sufrido unas mayores medidas de seguridad que recrudecen notablemente la experiencia carcelaria, ya sea a través del aislamiento o mediante la intensificación de las medidas como los recuentos y los cacheos (Fernández Abad, 2020).

pp. $9-26$

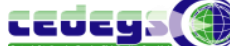


ENFOQUES JURIDICOS

REVISTA MULTIDISCIPLINAR DEL CEDECS

ISSN 26832070

Número 04

julio-diciembre 2021
Artículo: "La radicalización como elemento vertebrador de la política antiterrorista: una aproximación crítica"

María del Valle Cabeza Domínguez

Carlos Fernández Abad

DOI: https://doi.org/10.25009/ej.v0i04.2568

De este modo, parece evidente que, más que prevenir la participación futura del individuo en el fenómeno terrorista de una forma eficiente, el "discurso sobre la radicalización" favorece la criminalización de categorías enteras de población, concibiendo a los musulmanes -particularmente a los jóvenes inmigrantes de segunda y tercera generación- como una comunidad intrínsecamente sospechosa (Pantazis y Pemberton, 2009; Hickman, Thomas, Nickels y Silvestri, 2012; Breen-Smyth, 2014).

Además, resulta importante enfatizar que este adelantamiento de la barrera de intervención lleva aparejado la imposición de toda una serie de medidas de seguridad y control que, paradójicamente, contribuyen a acrecentar los sentimientos de vulnerabilidad y enfado que, desde este marco teórico, han sido señalados como una de las causas principales de este proceso. En estos términos, se podría llegar a plantear que este discurso no solo no es válido para prevenir de forma eficiente la participación del individuo en el fenómeno terrorista, sino que, además, también contribuye a crear las condiciones necesarias para que este se perpetúe.

\section{Conclusiones}

Tal y como se ha podido apreciar a lo largo de este trabajo, a pesar de su incuestionable hegemonía, la radicalización se presenta como un concepto especialmente problemático. $\mathrm{Si}$, de un lado, su naturaleza necesariamente relativa impide llegar a una definición universalmente aceptada sobre el término, del otro, tampoco existe un consenso claro sobre cuál debería ser considerado el punto final de este proceso, existiendo una importante discusión en la literatura especializada sobre si el hecho de abrazar unas ideas determinadas conduce inexorablemente al terrorismo. Además, según se ha argumentado, el "discurso sobre la radicalización" tiende a señalar como decisivas todo un conjunto de variables que, por su parte, también se encuentran presentes en grupos más amplios de población, lo que, a efectos prácticos, se traduce en que categorías enteras de población que nada tienen que ver con este fenómeno sean concebidas como potencialmente peligrosas. En este sentido, más que servir de base para la articulación de políticas públicas eficientes en esta materia, queda claro que la radicalización favorece la criminalización de grupos enteros de personas, contribuyendo de este modo a crear las condiciones que, paradójicamente, han sido señaladas como una de las causas de estos procesos.

Por todos estos motivos, parece evidente que, al menos por el momento, la lucha contra el terrorismo de inspiración yihadista no debería seguir estando principalmente sustentada sobre este concepto ya que, tal y como está formulado, favorece un adelantamiento de la barrera de intervención que, además de no estar justificado, facilita el desarrollo de actuaciones que resultan altamente lesivas y discriminatorias. Asimismo, en el campo específico del Derecho Penal, el "discurso sobre la radicalización" estimula la extensión de un modelo de respuesta que, orbitando en torno a la noción de excepcionalidad, trata a sus 
ENFOQUES JURÍDICOS

REVISTA MULTIDISCIPLINAR DEL CEDECS

ISSN 26832070

Número 04

julio-diciembre 2021
Artículo: "La radicalización como elemento vertebrador de la política antiterrorista: una aproximación crítica"

María del Valle Cabeza Domínguez

Carlos Fernández Abad

DOI: https://doi.org/10.25009/ej.v0i04.2568

destinatarios como enemigos cuya peligrosidad debe ser neutralizada de la forma más temprana posible, independientemente de si el valor de la acción realizada ha lesionado o puesto en peligro el bien jurídico protegido por la norma. Frente a ello y tomando en consideración el desdibujamiento de los límites del ius puniendi que esto implica, solo cabe postular la necesidad de transitar hacia un modelo que, estando basado en el garantismo penal (Ferrajoli, 1994), penalice únicamente aquellas conductas que resulten más graves y requieran de la intervención indispensable del Derecho Penal. 
ENFOQUES JURÍDICOS

REMISTA MULTIDISCIPLINAR DEL CEDECS

ISSN 26832070

Número 04

julio-diciembre 2021
Artículo: "La radicalización como elemento vertebrador de la política antiterrorista: una aproximación crítica"

María del Valle Cabeza Domínguez

Carlos Fernández Abad

DOI: https://doi.org/10.25009/ej.v0i04.2568

\section{Fuentes de consulta}

Alonso Pascual R. (2009). "Procesos de radicalización y reclutamiento en las redes de terrorismo yihadista". Cuadernos de Estrategia, 141, 21-68.

Antón Mellón, J. y Parra, A. (2015). “Concepto de radicalización”. En Antón Mellón, J. (ed.), Islamismo yihadista. Radicalización y contrarradicalización (17-37). Valencia: Tirant lo Blanch.

Baran, Z. (2005). "Fighting the War on Ideas". Foreign Affairs, 84 (6), 68-78.

Bartlett, J. y Miller, C. (2010). "The Edge of Violence: Towards Telling the Difference Between Violent and Non-Violent Radicalization". Terrorism and Political Violence, 24, 1-21.

Basra, R., Neumann, P. y Brunner, C. (2016). "The Edge of Violence: Towards Telling the Difference Between Violent and Non-Violent Radicalization". Terrorism and Political Violence, 24, 1-21.

Borum R. (2003). "Understanding the Terrorist Mindset". FBI Law Enforcement Bulletin, 7, 7-11.

Borum, R. (2012). "Radicalization into Violent Extremism I: A Review of Social Science Theories". Journal of Strategic Security, 4 (4), 7-36.

Breen-Smyth, M. (2014). "Theorising the suspect community: counterterrorism, security practices and the public imagination". Critical Studies on Terrorism, 7 (2), 223- 240.

Cano Paños, M. A. (2010). Generación yihad. La radicalización islamista de los jóvenes musulmanes en Europa. Madrid: Dykinson

Cassam Q. (2018). "The epistemology of terrorism and radicalisation". Royal Institute of Philosophy Supplements, 84, 187-209.

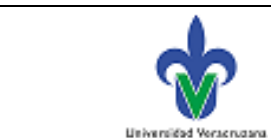


ENFOQUES JURIDICOS

REMISTA MULTIDISCIPLINAR DEL CEDECS

ISSN 26832070

Número 04

julio-diciembre 2021
Artículo: "La radicalización como elemento vertebrador de la política antiterrorista: una aproximación crítica"

María del Valle Cabeza Domínguez

Carlos Fernández Abad

DOI: https://doi.org/10.25009/ej.v0i04.2568

Coolsaet, R. (2019). "Radicalization: The Origins and Limits of a Contested Concept". En Fadil,N., Koning, M. y Ragazzi, F. (eds.) Radicalization in Belgium and the Netherlands. Critical Perspectives on Violence and Security (29-51). Londres: Tauris.

Crenshaw, M. (1982). "The Causes of Terrorism". Comparative Politics, 13 (4), 379-399.

Crone, M. y Harrow, M. (2011). "Homegrown Terrorism in the West". Terrorism and Political Violence, 23, 521-536.

Dalgaard-Nielsen, A. (2010). "Violent Radicalization in Europe: What We Know and What We Do Not Know". Studies in Conflict \& Terrorism, 33 (9), 797-814.

Della Porta, D. (1992). Social Movements and Violence: Participation in Underground Organizations. London: JAI Press.

Ferrajoli, L. (1994). Derecho y razón: teoría del garantismo penal. Madrid: Trotta.

Fernández Abad, C. (2020). "La doble problemática del terrorismo yihadista en prisión. Una aproximación crítica a la respuesta del sistema penitenciario español". Indret. Revista para el análisis del Derecho, 3, pp. 319-339.

Fernández De Mosteyrín, L.M. y P. Limón López P. (2017). “Paradigmas y prevención del terrorismo: una aproximación al Plan Estratégico Nacional de Lucha contra la Radicalización Violenta (PEN-LCRV 2015)". Política y Sociedad, 54 (3) 805-827.

Ghitens-Mazer, J. (2012). "The rhetoric an reality: radicalization and political discourse". International Political Science Review, 33 (5), 556-567.

Gobierno de España (2015). Plan Estratégico de Lucha contra la Radicalización Violenta (PEN-LCRV). Un marco para el respeto y el entendimiento común. Madrid: Secretaría de Estado de Seguridad.

Hafez M. \& Mullins C. (2015). "The Radicalization Puzzle: A Theoretical Synthesis of Empirical Approaches to Homegrown Extremism", Studies in Conflict \& Terrorism, 38 (11), 958-975.

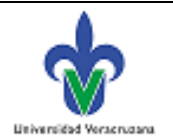

pp. $9-26$ 
ENFOQUES JURIDICOS

REMISTA MULTIDISCIPLINAR DEL CEDECS

ISSN 26832070

Número 04

julio-diciembre 2021
Artículo: "La radicalización como elemento vertebrador de la política antiterrorista: una aproximación crítica"

María del Valle Cabeza Domínguez

Carlos Fernández Abad

DOI: https://doi.org/10.25009/ej.v0i04.2568

Heath-Kelly, C. (2012). "Counter-Terrorism and the Counterfactual: Producing the Radicalisation Discourse and the UK PREVENT Strategy". The British Journal of Politics and International Relations, 15 (3), 1-22.

Hickman, M. J., Thomas, L., Nickels, H. y Silvestri, S. (2012). "Social cohesion and the notion of suspect communities: a study of the experiences and impacts of being suspect for Irish communities and Muslim communities in Britain". Critical Studies on Terrorism, $5(1), 89-106$.

Jenkins B. M. (2007) Building an Army of Believers: Jihadist Radicalization and Recruitment. Santa Monica, CA: RAND Corporation.

Jordán, J. (2009). "Procesos de radicalización yihadista en España. Análisis sociopolítico en tres niveles", Revista de Psicología Social, 2009, 24 (2), 197-216.

Kepel, G. (2006). The war of Muslim minds. Islam and the West. London: Harvard University Press.

Khosrokhavar, F. (2005). Suicide bombers. Allah's new martyrs. London: Pluto Press.

King M. y Taylor D.M. (2011). "The Radicalization of Homegrown Jihadists: A Review of Theoretical Models and Social Psychological Evidence". Terrorism and Political Violence, 23:4, 602-622.

Kundnani, A. (2012). "Radicalisation: the journey of a concept". Race \& Class, 54, 3-25.

Kundnani, A. (2015). A Decade Lost. Rethinking Radicalisation and Extremism. London: Claystone.

Lynch O. (2013). "British Muslim youth: radicalisation, terrorism and the construction of the other". Critical Studies on Terrorism, 6:2, 241-261.

Malthaner, S. (2017). "Radicalization. The Evolution of an Analytical Paradigm", European Journal of Sociology, 58 (3), 369-401.

McCauley C. y Moskalenko S. (2008). "Mechanisms of Political Radicalization: Pathways Toward Terrorism". Terrorism and Political Violence, 20:3, 415-433.

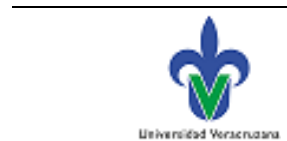


ENFOQUES JURIDICOS

REMISTA MULTIDISCIPLINAR DEL CEDECS

ISSN 26832070

Número 04

julio-diciembre 2021
Artículo: "La radicalización como elemento vertebrador de la política antiterrorista: una aproximación crítica"

María del Valle Cabeza Domínguez

Carlos Fernández Abad

DOI: https://doi.org/10.25009/ej.v0i04.2568

McCauley C. y Moskalenko S. (2014). "Toward a Profile of Lone Wolf Terrorists: What Moves an Individual from Radical Opinion to Radical Action". Terrorism and Political Violence, 26 (1), 69-85.

Moghaddam, F. M. (2005). "The Staircase to Terrorism: A Psychological Exploration". American Psychologist, 60 (2), 161-169.

Moyano, M. y Trujillo, H. (2013). Radicalización islamista y terrorismo. Claves psicosociales. Granada: Universidad de Granada.

Mythen, G., Walklate, S. y Peatfield, E. (2017). "Assembling and deconstructing radicalisation in PREVENT: A case of policy-based evidence making? Critical Social Policy, 37 (2), 180-201.

Neumann P. (2013). "The trouble with radicalization". International Affairs, 89:4, 873-893.

Neumann P. y Kleinmann S. (2013) “How Rigorous Is Radicalization Research?”. Democracy and Security, 9 (4), 360-382.

Pantazis, C. y Pemberton, S. (2009). "From the old to the new suspect community. Examining the Impacts of Recent UK Counter-Terrorist Legislation". British Journal of Criminology, 49, 646-666.

Paredes, J. M. (2020). “¿Para qué sirven las estrategias de prevención de la radicalización?”. En Fernández Abad, C. y González León, C. (coord.). La RES 2178 de NU y su trasposición a los derechos penales nacionales. Propuestas de equilibrio entre la seguridad y los derechos individuales (201-224), Navarra: Thomson Reuters Aranzadi.

Pérez Cepeda, A, I. (2018). "Política criminal frente a la radicalización de menores de extrema derecha: ¿un modelo más racional?”. En Alonso Rimo, A., Cuerda Arnau, M. L., Y Fernández Hernández, A. (Dir.) Terrorismo, sistema penal y derechos humanos (649-676), Valencia: Tirant lo Blanch.

Pisoiu D. (2012). Islamist Radicalistion In Europe. An occupational change process. Reino Unido: Routledge.

pp. $9-26$

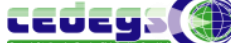


ENFOQUES JURÍDICOS

REVISTA MULTIDISCIPLINAR DEL CEDECS

ISSN 26832070

Número 04

julio-diciembre 2021
Artículo: "La radicalización como elemento vertebrador de la política antiterrorista: una aproximación crítica"

María del Valle Cabeza Domínguez

Carlos Fernández Abad

DOI: https://doi.org/10.25009/ej.v0i04.2568

Rabasa, A., Pettyjohn, S., Ghez, J. y Boucek, C. (2010). Deradicalizing Islamist Extremists. Santa Mónica: RAND Corporation.

Reinares F. y García Calvo C. (2012). “¿Cómo evitar los procesos de radicalización yihadista en nuestra sociedad?: consideraciones sobre la experiencia británica". Real Instituto Elcano, ARI 188/2012, 1-6.

Reinares F., García-Calvo C. y Vicente A. (2019). Yihadismo y Yihadistas en España. Quince años después del 11-m. Madrid: Real Instituto Elcano.

Richards, A. (2011). "Conceptualizing Terrorism". Studies in Conflict \& Terrorism, 37, 213236

Roy, O. (2005). El islam mundializado. Barcelona: Bellaterra.

Roy, O. (2008). "Al Qaeda in the West as a Youth Movement: The Power of a Narrative". Centre for European Policy Studies, 168, 1-8.

Sageman M (2008). "A Strategy for Fighting International Islamist Terrorists". The ANNALS of the American Academy of Political and Social Science, 618 (1), 223-231.

Sageman, M. (2004). "Understanding Terror Networks". Philadelphia: University of Pennsylvania, 2004.

Sedgwick M. (2010). "The Concept of Radicalization as a Source of Confusion". Terrorism and Political Violence, 22, 479-494.

Silber M. y Bhatt A. (2007). "Radicalization in the West: The Homegrown Threat". Nueva York: New York Police Department Intelligence Division.

Veldhuis, T. y Staun, J. (2009). "Islamist Radicalisation: A Root Cause Model". The Hague: Netherlands Institute of International Relations Clingendael.

Wiktorowicz, Q. (2006). "Radical Islam Rising. Muslim Extremism in the West". Lanham: Rowman \& Littlefield.

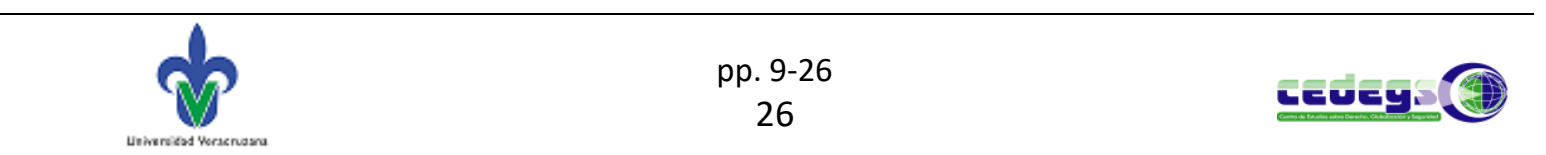

